\title{
Distributed Processing and Analysis of Physics Data in the D0 SAM System at Fermilab
}

\author{
Igor Terekhov \\ Fermi National Accelerator Laboratory, Batavia, IL 60510 \\ terekhov@fnal.gov
}

May 4, 2001

\begin{abstract}
SAM (Sequential Access through Meta-data) is the data access system for the D0 high energy physics (HEP) experiment at Fermilab. The system is being developed and used to handle the Petabyte-scale experiment data. The D0 applications, like virtually all HEP applications, are data-intensive, which poses special problems for the data management and job control facilities in the distributed environment. The fundamental problem is to bring the user applications and the data together, and SAM attacks the problems from both sides. First, we describe how the system moves the data through the distributed disk cache. Second, we describe how SAM interacts with the batch system to synchronize parallel user jobs with the data availability. All the design solutions herein have been implemented in a real system that handles the mission-critical data of the D0 experiment; thus, we present our work from the standpoint of real experience.
\end{abstract}

KEYWORDS: data-intensive applications, distributed systems, parallel processing

\section{Overview}

In the past, most scientific jobs, including those at the D0 experiment at FNAL, were run and controlled by the batch systems. The classic batch system is however, strongly centered at computing resources and does not take into account the data availability, particularly when the data is to be retrieved from outside the processing station. Scientists were responsible for writing pre-requisite job scripts that would ensure, on a per user basis, the availability of the data prior to the job dispatch. There has not been a provision to run long jobs whose 
input data size exceeds the capacity of a local station that would require data replenishment in the course of the job run. Unless it could be described by e.g., a single file, input data was never part of the job specification, which made it impossible to coordinate usage of resources connected with data movement across multiple jobs, and data sharing was minimal.

Aside from being inconvenient for the users and error-prone, the old approach is simply impossible with the modern amounts of data and access volumes. In Run II, which is a major upgrade of the accelerator facility at Fermilab, the D0 experiment will accumulate on the order of 1 peta-byte of data whose significant fraction will continuously be accessed by hundreds of collaborators throughout the world. Furthermore, the HEP community moves rapidly towards distributed computing whereby collaborators prefer owning of highly efficient locally operated clusters to committing resources to a central power.

In response to these and other challenges, the D0 experiment is developing a fully distributed data handling system, SAM. It is an ongoing project[1], which has a number of goals, or aspects, more fully described elsewhere[2, 3]: full data cataloging, including processing history and dataset management, globally distributed uniform access by possibly remote users, data replication and caching on disk, hardware resource management and user job control. In the center of the system is the meta-data (the "M" in SAM) catalog implemented in a relational database (Oracle). Most of the data model of the catalog pertains to the contents description of the actual physics data, which is somewhat D0-specific and is left outside of this paper's scope. Other meta-data is used for the actual data-handling system and include data replica catalog (file location tracking), resource management policies and configuration information. The actual data movement and job management is done by a hierarchy of servers communicating via CORBA[9] interfaces among themselves and with the database. The complete description of the architecture is found in various documents at the SAM WWW site[1]. The system has been operational in production use for over two years, with much practical experience gained in the time.

In our paper we concentrate on how the data is distributed and processed in parallel fashion with SAM. The data access is fully distributed in the sense that the hardware resources are not concentrated on any particular machine or even site, rather, they are arbitrarily apportioned by the processing stations. Each station is typically a homogeneous cluster or a single multi-processor machine. A server called station master runs at each station to coordinate the associated collection of resources, as well the user jobs to be run therein. The system is therefore distributed in both the global (the network of stations) and the local (within a station) senses. In the present paper we concentrate on the local cluster aspects; some of the global aspects are described in [4], others constitute future work we outline at the end of the paper. We first describe the physical data management, which for the reasons explained later is essentially the distributed disk caching for the purposes of "bringing the data to the 
applications". Second, we describe the batch system integration component, which is responsible for communication between the data delivery system with the abstract batch system, for the purposes of "bringing the applications to the data".

\section{Disk Caching}

In the earlier days, the D0 experiment envisioned that the bulk of the data would be stored on tape in a mass storage system (MSS) and that the primary purpose of disk caching would be to maintain a dynamically selected small subset of data on disk for user access, thus mediating user access to the $\mathrm{MSS}^{1}$. Access to an MSS is usually expensive because it often requires operator mount or mechanical robotic arm movement, with latencies far exceeding computer data access time scale. The SAM architecture therefore included a global optimizer primarily to control MSS access (not discussed here).

As we have already said earlier, for a lack of the distributed technologies and infrastructure, data access (especially analysis) tended to be centralized on large cost-inefficient analysis servers. Such centralized servers, while accumulating large disk by most traditional measures, could not always handle any significant fraction of the data (e.g., for about one peta-byte of the total D0 data, only a few tera-bytes of disk was initially envisaged).

With the de-centralization of the data access at D0, the mission of the disk data caching in SAM is transforming into taking full advantage of the distributed network of disk caches. A significant subset of the interesting data can in fact be placed on such distributed disk and replicated as necessary often without the costly MSS access. In this section we show how the SAM architecture handles the challenge of distributed caching.

Each machine in a cluster at a station has locally mounted disks. These disks are known to the station master and are part of its configuration, stored in the aforementioned database. Running at each machine is a station's agent called stager (the name is due to the fact that making a local copy of data is called staging in HEP). The stager has physical access to the local file systems and invokes file transfer facilities. The latter are selected based on the type of transfer; for MSS access the MSS-specific client is invoked whereas for disk to disk copies an efficient ftp-like program such as bbftp is used.

When the station's cache manager receives a request to cache a dataset, it issues a request to the "global" (site-wide) optimizer mentioned above (which has evolved from an MSS access controller to a manager of network traffic and other global resources). Requests for different datasets are regrouped based on global resource optimization consideration. For every group of files authorized for transfer, the cache manager allocates disk space from the distributed pool

\footnotetext{
${ }^{1}$ The D0 MSS of choice is the Enstore system[5].
} 
of caches on the cluster and issues requests to the stagers to deliver the group at about the same time.

Cache replacement is based on the file usage. The physical total disk of the station is logically divided by groups, each group using its own cache management scheme. Multiple cache management algorithms (such as LRU, FIFO, various flavors thereof, etc.) are known and configured at run time in the station independently for each group, as in the "strategy" design pattern. When data used by different groups have little overlap, having multiple independent virtual cache managers gives great flexibility in disk usage. When different groups access some files, the files' ownership migrates accordingly after the last or most frequent user. The tracked "users" of the cached files are not the user applications, these are the projects to be described later in the job management section.

We will note in passing that, while managing the locally distributed caches, stations exchange data in the course of global data routing and replication as we described earlier in [4].

\section{Job Management}

In the present discussion, by user job we primarily mean the activity of computation on data that has been retrieved and cached by the above cache manager. Production and storing of data by user applications is largely symmetric to the consumption. The user job specification, when submitted to the system, therefore contains two parts: the application to be run (e.g., a script or a binary name), and the input dataset ${ }^{2}$. At present, the job is submitted to a station selected by the user, although we envision a global request broker in the future to balance the load among the stations.

The end goals of the job control and data management are to ensure "fair" resource allocation by certain user categories, subject to the collaboration's policies, and maximization of the overall job throughput. In the data intensive world, both goals require approaches that fully incorporate the data management issues. To attain the first goal, the system uses fair share scheduling; for the second, the system performs resource co-allocation.

\subsection{Fair Share Allocation and Scheduling}

The SAM scheme for Fair Share Allocation and Scheduling (FSAS) of resources and user jobs, respectively, serves more than the purpose of data consideration in job scheduling. It provides a flexible and general framework that can be used in any job scheduling environment.

\footnotetext{
${ }^{2}$ Although specification of the intended output is desired, the output is seldom predictable before the job is run and is absent initially.
} 
For the purposes of FSAS, the system uses the concept of a "benefit". Whenever a user job is run, the user ${ }^{3}$ receives a number of benefits of different types, because it (a) uses or consumes resources, and (b) accomplishes some (hopefully useful) results. Each resource is assigned a benefit type; in addition, having achieved certain work, such as having processed an input file, also counts as a benefit. In fact, any quantifiable quantity that increases monotonically with every job run may be treated as a benefit type. A cumulative vector of benefits received by each user is maintained. At the time of job submission, and possibly at run time, the user's fraction of the cumulative benefits in the grand total benefit allocation for all users, is computed. The fraction received by this user is compared to its allotted fraction of benefits. To do so, the station master weights the different components of the benefit vector by configurable quantities called benefit type weights. If the user is below its intended fair share, its job will be scheduled ahead of the other jobs, and vice versa.

Note that simpler versions of fair share scheduling are used in some batch systems. For example, the LSF commercial batch system uses CPU consumption alone to schedule jobs. In our system, on the other hand, the administrators are given the power to control which benefit types contribute heavier into the fair share scheduling, thus focusing on the resources that are most scarce, or, under the overall shortage of resources, guaranteeing that the amounts of work done by different users reflect the democratically decided priorities and policies.

\subsection{Co-allocation of Data Management and Computing Resources}

When a user job is submitted to the station master, the latter translates the input dataset into an explicit set of files using the meta-data catalog mentioned in Sec. 1. The already available files, if any, are "locked" temporarily for the duration of the project being started. The station master launches the project master (Sec. 3.4) and queues data retrieval for the files missing from the caches, if any. The cache replenishment for this project, as part of the overall station cache management described above, is done asynchronously from the progress of the job, to minimize the overall job turnaround time. The initial dispatch of the job is, however, coordinated with the availability of some minimum amount of data (usually, any one file from the dataset) on local disks. The SAM system augments the original user job specification by additional (user-transparent) requirement of a certain resource becoming available, or an event occurrence. If there are no files available to the job at all, the job will not be dispatched until SAM signals somehow to the Batch System (BS) that the job is runnable. SAM thereby brings jobs to the data in the temporal sense.

\footnotetext{
${ }^{3}$ In our resource management discussion, a "user" is usually a group of individual users, so that resources are allocated by groups rather than by individuals.
} 


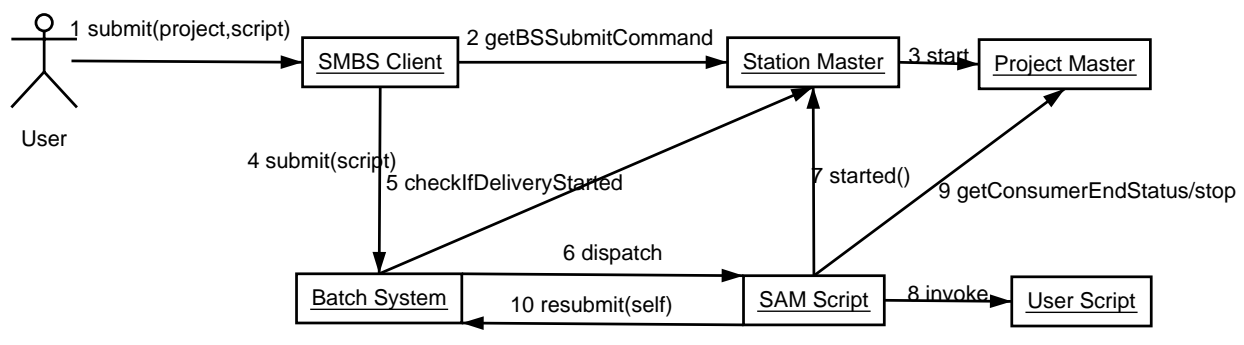

Figure 1: Station Master - Batch System (SMBS) Collaboration Diagram.

SAM also strives to bring the applications to the data in the spatial sense, contingent on the capabilities of the underlying BS. In a distributed environment on a cluster, when some dataset files are already cached, the SAM system will "recommend" to the BS where the job's processes should be dispatched. Note that this recommendation cannot be a directive because in general, the initial availability of the files may not be the most important criterion for the selection of the execution sites, even in the data-intensive environment (Sec. 4).

\subsection{Implementation of the Data Delivery Integration with the Batch System}

The above design ideas are carried out as follows, see Fig. 1. The client passes the input dataset specification to the SM which uses the above considerations to return the exact job submission command string. The exact mechanisms for communicating information between SAM and the Batch System (BS) are dependent on the batch system in question, of course, that is why SAM uses abstract BS adapters to execute the above plans. For example, in one batch system the job is made to wait on data arrival by requiring a resource that is coded as the name of the job's project. In another, the batch system awaits an explicit event for the job. The SAM system will declare resource availability in the first case and generate event in the second. All the details about the particular batch systems are hidden into the respective adapters.

The user job is wrapped, also transparently to the user, into a SAM script that finally invokes the user script. The wrapper script is capable of resubmitting itself when a long job fails on account of temporary data unavailability. In addition, the wrapper script ensures proper clean-up and benefit accounting, Sec. 3.1.

Note that in the Figure, the initial data availability is polled by the batch system, e.g., by asking for an abstract resource or executing an external preexecution command. Some BS's require that such an event is in fact pushed into the BS, which is supported as well.

For the purposes of this paper's relevance to data-intensive computing in 
general, it is very important that the data delivery system in SAM and the Batch System are coupled rather loosely. Virtually any BS can be adapted to our scheme, and, symmetrically, any external data delivery system may be interfaced with the BS as our scheme suggests.

\subsection{Actual Data Processing}

During the run of the job, or "project", consumption of files by applications is coordinated by the server called project master. The details of this have been described earlier, see [6]; we merely summarize the scheme here. The D0 applications served by the system process and/or analyze HEP data, which is typically a collection of events objects. SAM caches the data in the form of files on the local disk, with applications having the infrastructure to deliver event objects to the end user code[7]. Some D0 applications are readily parallelizeable, i.e., allow multiple threads or processes, others require users to do special work to combine results. Parallelism infrastructure in the application is outside the scope of SAM, however, and the applications need not be D0 applications in the strictest sense. The project master in SAM will ensure distribution of files (cached for it by the station master) to the consumer processes al long as they conform to a simple protocol.

\section{Future Work}

The future design work in SAM is planned for the overall resource management and job control, with the emphasis on global distribution. One aspect of particular importance is studying and balancing the relative costs of dispatching a job at a site with computing resources, but without a priori available data, versus queuing the job at a "busy" site where the data is available. Already at a small distributed cluster, it is evident that the initial availability of (some) of the project's data at a machine is only one of the several factors to affect the job placement. The sites recommended by the cache manager may or may not be used by the job scheduling system, depending on the "costs" of data replication and the "value", or "benefit" of running the job sooner rather than later.

We therefore plan to develop a general language to express resources as well as "costs" and other economics-like terms, in order to address such issues, especially with the inter-cluster, inter-station job management. This work is planned for a collaboration with Computer Scientists in the course of the Particle Physics Data Grid, most notably with the Condor team[8]. 


\section{Acknowledgements}

This work presents some of the conceptual design of the SAM project. The work is only a part of the project, carried out by the team of several people [1], who design, implement, integrate, test and support the system. The author is proud to be a member of this group. The author is grateful to Ruth Pordes and

Gabriele Garzoglio of FNAL for comments on the content and quality of this paper.

\section{References}

[1] The SAM team, L.Lueking (co-leader), V.White (co-leader), L. LoebelCarpenter, C. Moore, H.Schellman, I.Terekhov, J.Trumbo, S.Veseli, M.Vranicar. The home page http://d0db. fnal.gov/sam.

[2] L. Carpenter et. al. "SAM Overview and Operation at the D0 Experiment", submitted to The International Conference on Computing in High Energy and Nuclear Physics (CHEP 2001), September, 2001.

[3] V.White for the SAM project, "The Data Access Layer for D0 Run II: Design and Features of SAM", talk given at The International Conference on Computing in High Energy and Nuclear Physics (CHEP 2000), February, 2000, Padova, Italy,

[4] I. Terekhov et. al. "SAM for D0 - a fully distributed data access system", talk presented at VII International Workshop on Advanced Computing and Analysis Techniques in Physics Research (ACAT 2000), Oct. 2000, Batavia, IL, in proceedings.

[5] The Enstore home page http://www-isd.fnal.gov/enstore.

[6] I. Terekhov and V. White, Distributed Data Access in the Sequential Access Model at the D0 Experiment at Fermilab, poster presentation, in proceedings of The Ninth IEEE International Symposium on High Performance Distributed Computing, Aug, 2000, Pittsburgh, PA

[7] The D0 framework is described by J. Kowalkowski, in "The D0 Framework", talk given at CHEP2000, see [3].

[8] The Condor project home page http://www.cs.wisc.edu/condor/.

[9] The OMG home page http://www.omg.com. For C++ components, "ORBacus for $\mathrm{C}++$ and Java" http://www.ooc.com. For Python components, "Fnorb, a Python ORB" http://www.fnorb.org. 